\title{
LITHIUM IN POPULATION II STARS
}

\author{
Not a Perfect Window into the Big-Bang
}

\author{
J.A. THORBURN \\ Yerkes Observatory, University of Chicago \\ P.O. Box 258, Williams Bay, WI 53191 USA
}

\begin{abstract}
Since the work of Spite \& Spite (1982), halo main sequence turn-off stars have usually been considered a site where Li synthesized in the Big-Bang is wholly preserved and uncontaminated by other Li sources. However, the interpretation of halo dwarf Li abundances has become more complex as new samples of halo star Li I observations have become available. Linking the Li plateau abundance identically with the cosmological Li fraction is no longer warranted. At present, observational evidence favors a scenario where most-but not all-of the plateau abundance is cosmological in origin and a non-negligible amount is contributed subsequently by Galactic nucleosynthesis; severe, uniform Li depletion is constrained but not yet completely ruled out.
\end{abstract}

\section{The Spite Plateau}

The first observations of the Li I $\lambda 6707$ doublet in halo stars were reported by Spite \& Spite (1982). Contrary to conventional wisdom, which held that Li should not survive in the oldest stars, the Spites found that stars with $[\mathrm{Fe} / \mathrm{H}]<-1$ and solar-like effective temperatures $6300<\mathrm{T}_{\text {eff }}<5600$ $\mathrm{K}$ show indistiguishable $\mathrm{Li}$ abundances within an estimated observational error of \pm 0.15 dex. A comparable sample of field Population I stars, in contrast, show a two order of magnitude range of $\mathrm{Li}$ abundances. The extraordinarily small scatter in $\mathrm{N}(\mathrm{Li})$ observed in halo stars, despite a range in mass, chemical content, and diverse Galactic orbits, suggested that the $\mathrm{Li}$ on the surfaces of halo stars could reflect the unaltered abundance of $\mathrm{Li}$ produced in Big-Bang nucleosynthesis (BBN). 


\section{New Developments}

Recently, observational and theoretical evidence which challenges the simplest interpretation of the Spite plateau has come to light. Three extremely metal-poor dwarfs with temperatures above $5900 \mathrm{~K}$ show $\mathrm{Li}$ abundances a factor of ten or more below the plateau (Thorburn 1992). Models of rotating stars show that all halo stars are hypothetically capable of depleting their original Li by an order of magnitude or more (Pinsonneault et al. 1992; Chaboyer 1993). Tests of this prediction using tidally-locked halo binaries have been inconclusive, except among cooler halo stars (Ryan \& Deliyannis 1994). A re-examination of literature halo star Li data (Deliyannis et al. 1993) and a new, large sample of Li data (Thorburn 1994) each indicate greater scatter in Li I feature strengths at a given metallicity and dereddened color than that produced by known causes of color scatter. A weak correlation between $\mathrm{N}(\mathrm{Li})$ and $[\mathrm{Fe} / \mathrm{H}]$ has been observed among plateau stars with $[\mathrm{Fe} / \mathrm{H}]<-2$ (Thorburn 1994; Norris et al. 1994); minor Galactic Li production is the probable cause. A slight positive slope of the $\mathrm{N}(\mathrm{Li})$ vs. effective temperature relation has also been noted (Thorburn 1994) but may reflect a combination of non-LTE effects and systematic errors in the effective temperature scale rather than a physical dependence of $\mathrm{N}(\mathrm{Li})$ on temperature. The more delicate isotope ${ }^{6} \mathrm{Li}$ has also been detected at the 5\% level in HD 84937 and, provisionally, HD 201891 (Smith et al. 1993; Hobbs \& Thorburn 1994). As ${ }^{6} \mathrm{Li}$ is not produced in appreciable amounts during standard BBN, Galactic contamination is the likely cause.

Observational evidence no longer supports the original interpretation of the Spite plateau: the Big-Bang Li fraction is not identical to the surface $\mathrm{Li}$ abundances of the plateau stars. The simplest scenario which does account for the data requires $\sim 70 \%$ of halo star Li to be produced in standard BBN and modest Galactic Li production to contribute the remainder (Thorburn 1994). However, the extremely Li deficient stars continue to defy explanation.

\section{References}

Chaboyer, B.: 1993, Ph.D. thesis, Yale University.

Deliyannis, C.P., Pinsonneault, M.H., and Duncan, D.K.: 1993, Astrophys. J., 414, 740.

Hobbs, L.M. and Thorburn, J.A.: 1994, Astrophys. J. Lett., 428, L25.

Norris, J.E., Ryan, S.G., and Stringfellow, G.S.: 1994, Astrophys. J., 423, 386.

Pinsonneault, M.H., Deliyannis, C.P., and Demarque, P.: 1992, Astrophys. J. Suppl., 78, 179.

Ryan, S.G. and Deliyannis, C.P.: 1994, Astrophy. J., in press.

Smith, V.V., Lambert, D.L., and Nissen, P.E.: 1993, Astrophys. J., 408, 262.

Spite, F. and Spite, M.: 1982, Astron. Astrophys., 115, 357.

Thorburn, J.A.: 1992, Astrophys. J. Lett., 399, L83.

Thorburn, J.A.: 1994, Astrophys. J., 421, 318. 